\title{
Surgical extraction of migrated coils via proximal segment of the anterior cerebral artery: An emergency alternative
}

\author{
Zhi Chen, Weihua Tang, Hua Feng, Gang Zhu \\ Department of Neurosurgery, Southwest Hospital, Third Military Medical University, Gaotanyan Street, Shapingba District, \\ Chongqing - 400 038, China
}

\author{
Address for correspondence: \\ Dr. Gang Zhu, \\ Department of Neurosurgery, \\ Southwest Hospital, Third Military \\ Medical University, Gaotanyan \\ Street, Shapingba District, \\ Chongqing - 400 038, China. \\ E-mail: a65427851@cta.cq.cn
}

DOI: $10.4103 / 0028-3886.53286$

\begin{abstract}
Coil migration is a severe complication in endovascular treatment. We report our experience of prompt surgical extraction of migrated coils via excised proximal AI following endovascular embolization of intracranial aneurysms in two patients. Both patients had to undergo coiling or stent-assisted coiling for cerebral aneurysms, and while operating the coils protruded and migrated to the bifurcation of internal carotid artery and middle cerebral artery. Immediate craniotomy was performed after unsuccessful or unfeasible endovascular retrieval. The migrated coils were removed via excised proximal $\mathrm{Al}$, and the patients recovered well without any neurological deficits. We suggest that this surgical procedure might be an alternative route in selected patients with good cross circulation in emergency. In addition, a rare occasion of coil migration after stent-assisted coiling is also described in one patient, which might have resulted from unsatisfactory wall apposition of the stent due to a curved vessel.
\end{abstract}

Key words: Coil migration, embolization, intracranial aneurysm, stent

\section{Introduction}

Coil protrusion or migration into the parent artery is one of the most feared complication in endovascular treatment, in which neurovascular surgery should be reserved as an appropriate treatment alternative. ${ }^{[1-4]}$ In this report, we describe our experience of prompt surgical extraction of migrated coils via proximal A1 segment.

\section{Case Reports}

\section{Case 1}

A 61-year-old female presented with acute subarachnoid hemorrhage (SAH) and her clinical status was Hunt and Hess grade II on admission. Angiogram revealed a wide-necked aneurysm of the left posterior communicating artery [Figure 1a]. Endovascular treatment was then performed under general anesthesia and systemic heparinization. For this patient, we used
EDC (EV3/ Micro Therapeutics, USA) for aneurysmal coiling. The coil mass seemed stable within the aneurysm sac after the first complex coil $(6 \mathrm{~mm} \times 7 \mathrm{~cm})$ was placed. Immediately after detachment of the second complex coil $(5 \mathrm{~mm} \times 6 \mathrm{~cm})$, most of the coil mass, together with the microcatheter, prolapsed into the internal carotid artery (ICA) [Figure 1b]. Subsequent unsuccessful attempts were made to retrieve the migrated coils using microsnare. Contralateral ICA angiogram revealed the coil mass had migrated into the more distal ICA and middle cerebral artery (MCA), causing obvious flow compromise of the left MCA [Figure 1c]. In this circumstance, the patient was transferred immediately to the operating room for emergency surgery. A left pterional craniotomy was performed. The aneurysm was found following dissection of the sylvian fissure. The coils visible through the vessel had totally migrated out of the aneurysm and into the ICA bifurcation and the MCA. After the placement of temporary clips, the dome of the aneurysm was incised. Attempts to 
extract the coils from the incised aneurysm were futile, following which the aneurysm was clipped. Considering that the main part of coil mass was located within the ICA bifurcation, extraction of the migrated coil via proximal segment of the anterior cerebral artery (ACA) was chosen. The proximal ACA was cut at $3 \mathrm{~mm}$ from the bifurcation with microscissors, and the coils were removed with a blunt microhook. Microvascular Doppler demonstrated good flow in the left ICA and MCA. The patient recovered from anesthesia with mild and transient hemiparesis in her right upper limb (grade IV). Postoperative CT angiogram showed patency of the both A2 and MCA [Figure 1d]. At the one-year follow- up evaluation, the patient was neurologically intact.

\section{Case 2}

A 49-year-old female presented with three months of dizziness. The patient underwent $\mathrm{CT}$ angiogram in a local hospital, which revealed a right unruptured paraclinoid aneurysm. On her admission to our hospital, physical examination showed no exact neurological deficits. Right ICA angiogram showed a wide-necked paraclinoid aneurysm [Figure 2a]. Stent-assisted coiling was chosen for the unfavorable dome- to-neck ratio of the aneurysm. The patient received double antiplatlet medications, including $100 \mathrm{mg}$ of aspirin and $75 \mathrm{mg}$ clopidogrel, daily for three days before stent deployment. Endovascular treatment was then performed under local anesthesia and systemic heparinization. For this patient, we used LEO stent (BALT, France) and MicroPlex coil (Microvention, USA) for treatment. Standard methods of stenting and trans-stent coil delivery were used as described in the literature. ${ }^{[5,6]}$ Firstly, a LEO stent of $4 \times 15 \mathrm{~mm}$ was successfully deployed across the neck of the aneurysm. Subsequent aneurismal coiling was performed after the microcatheter was navigated through the interstices of the stent into the aneurysmal sac. Angiogram after
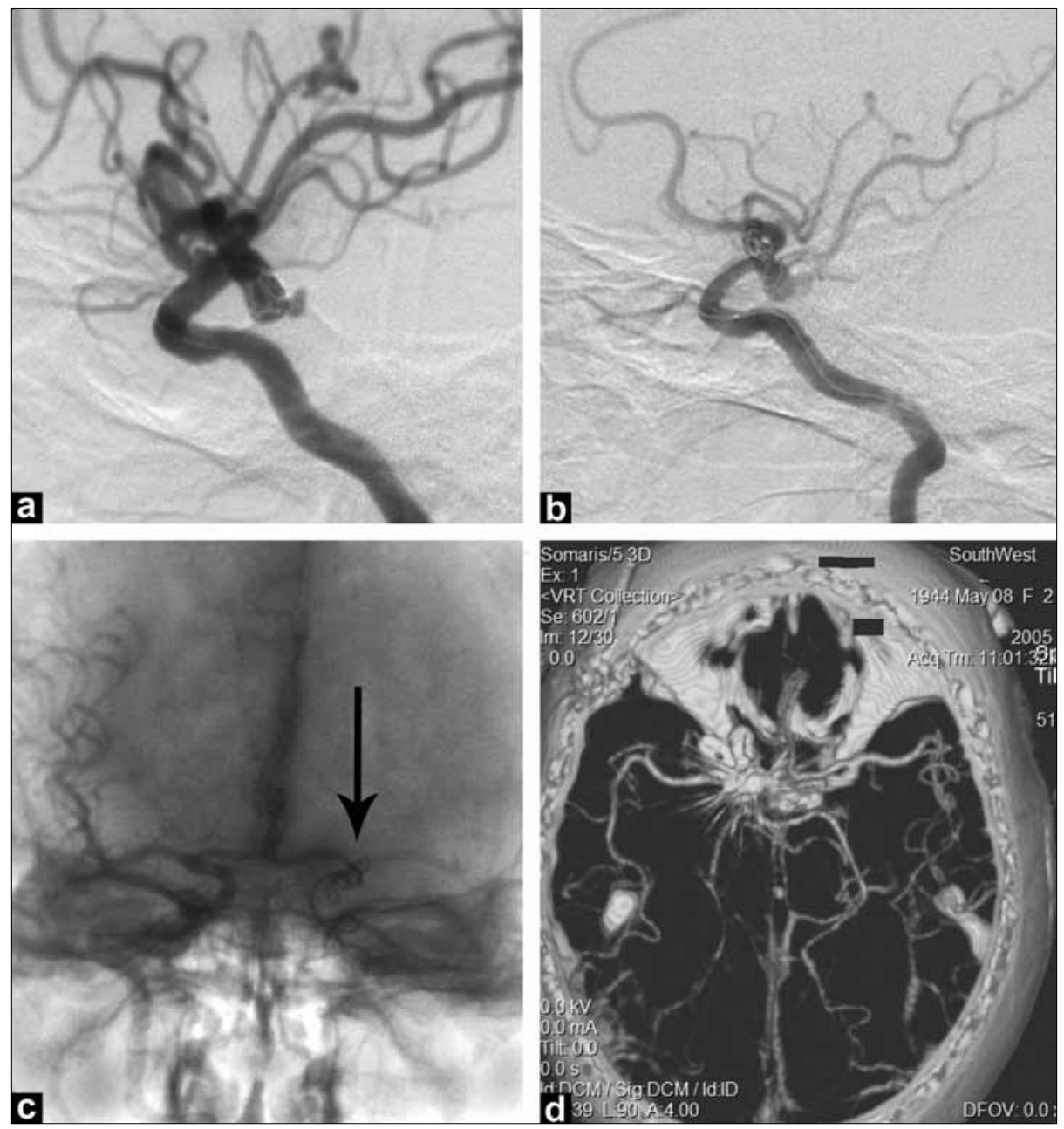

Figure 1: (a) Left internal carotid artery angiogram showing a wide-necked aneurysm of the left posterior communicating artery, and the coil mass seems stable after placement of the first complex coil; (b) Angiogram after inserting the second coil showing most of the coil mass, together with the microcatheter, prolapsed into the parent artery; (c) Subsequent unsubtracted angiogram of the right internal carotid artery showing the migration of the coil mass to distal internal carotid artery and middle cerebral artery, causing obvious flow compromise of the left middle cerebral artery; (d) Postoperative CT angiogram showing the patency of both the A2 and middle cerebral artery 
detachment of the second coil showed part of the coil mass protruding into the ICA artery without obvious flow reduction [Figure 2b]. Repeated angiogram 30 min utes later showed no obvious change of the coil mass compared with the previous angiogram, and the patient was neurologically intact. The patient was transferred to the intensive care unit for close follow up. Skull plain radiograph performed a day later revealed that the coil had migrated distally into the MCA [Figure 2c]. Considering the difficulties to retrieve the coils through the stent by endovascular procedure, we decided to remove the migrated coil surgically. As illustrated in Case 1, coil extraction from the proximal ACA following aneurysm clipping was done via a right pterional approach. The patient made a good recovery from surgery. Postoperative CT angiogram at one week showed the patency of both the A2 and MCA [Figure 2d]. At the 10-month follow-up evaluation, the patient was neurologically intact.

\section{Discussion}

Coil protrusion or migration into the parent artery has been known to be one of the main complications in the coil embolization of cerebral aneurysms, which might result in severe neurological deficits. ${ }^{[1-4,7]}$ Compared with coil embolization alone, coil migration from the confinement of the stent is a rare event. ${ }^{[6,7]}$ Small coils together with $2 \mathrm{~F}$ stent cells were thought to play a role in the escape of a coil from the confinement of a stent. ${ }^{[6]}$ However, we believed Case 2 in our report represented a different situation. The LEO stent used in this case is made of nitinol-twisted wires in a closed-cell design. Though closed-cell design stents such as LEO stent will maintain a constant cell opening at the convexity of a vessel, it has, in principle, less flexibility to conform to a curved or irregular anatomy. It has recently been observed in a curved vessel model that the LEO stent showed an inward crimping of the proximal and distal
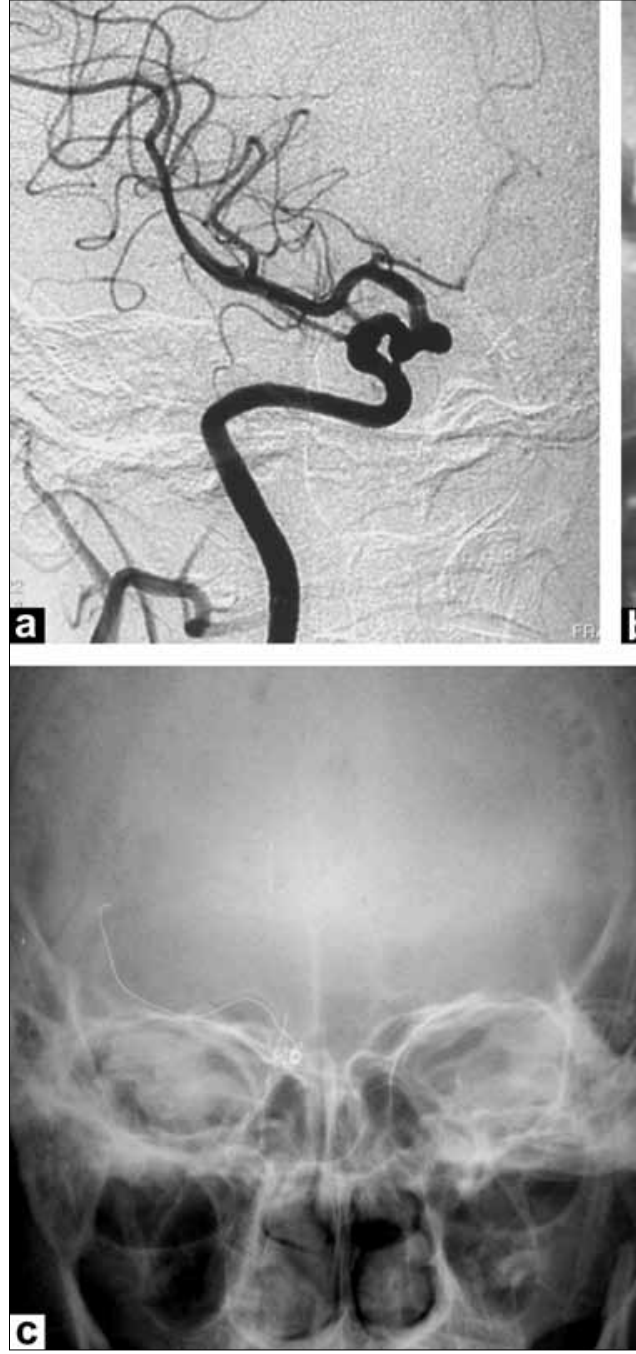
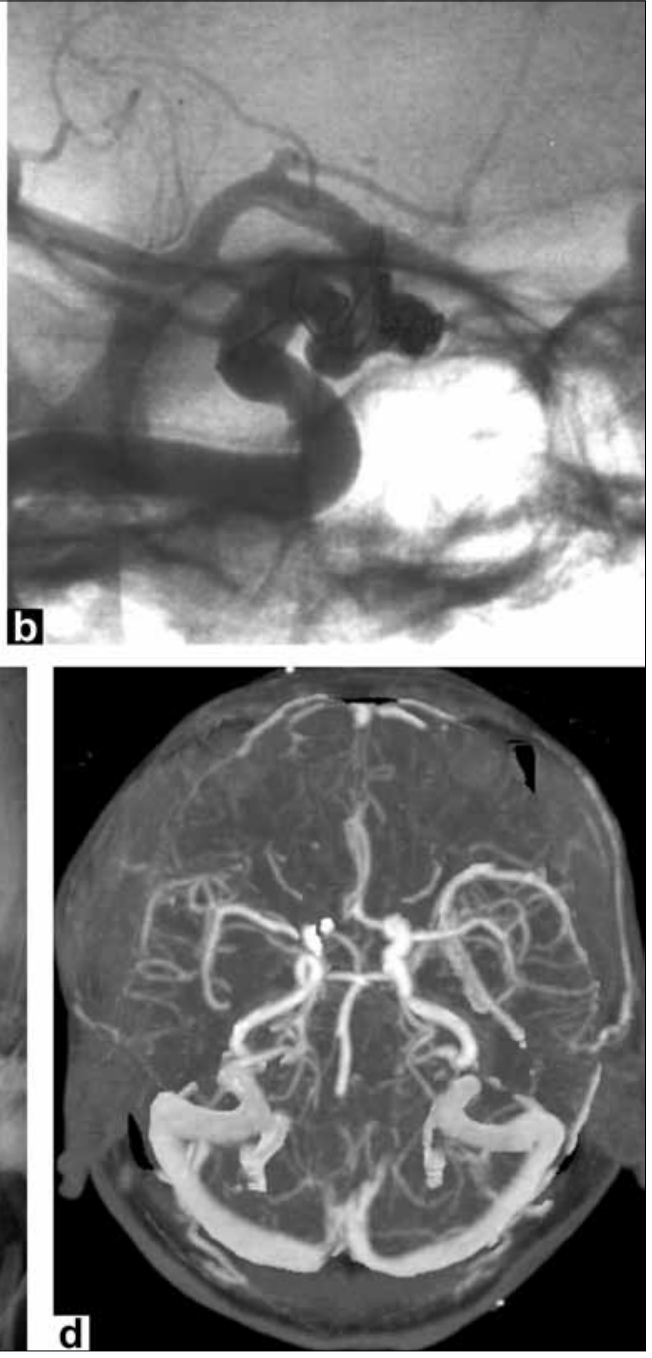

Figure 2: (a) Right internal carotid artery angiogram showing a wide-necked paraclinoid aneurysm; (b) Angiogram immediately after stent-assisted coiling demonstrating incomplete occlusion of the aneurysm. Part of the coil mass protruding into the internal carotid artery lumen without obvious flow reduction; (c) Plain radiograph in the anterioposterior view one day after the embolization showing the coil stretched and migrated into the middle cerebral artery; (d) Postoperative CT angiogram showing the patency of both the A2 and middle middle artery 
ends, significantly narrowing the lumen of the stent at these points. ${ }^{[7]}$ Therefore, we believe that the tortuous and curved ICA siphon in our Case 2 might have lead to the unsatisfactory wall apposition of the stent to the ICA lumen, and the coil mass protruding into the ICA lumen because of the narrowing of the effective stent lumen.

Regarding removal of the migrated coils, several endovascular devices and techniques have been used to retrieve them. ${ }^{[8]}$ In addition, stent placement was also reported to reconstruct the arterial lumen past a migrated coil. ${ }^{[9]}$ Nevertheless, endovascular retrievals or deployment of a stent are not always feasible. Besides, further endovascular procedures may risk pushing the migrated coil more distally, resulting in a more complex situation. Therefore, open surgery is still required when endovascular procedures are difficult or unsuccessful. ${ }^{[2-4]}$ When the coil is still partly within the aneurysm, removal of the migrated coil by opening the dome of aneurysm is relatively easy. ${ }^{[1]}$ There were only a few reports ${ }^{[2-4]}$ regarding the surgical procedures for distally migrated coils that could not be extracted from the incised aneurysm sac. Deshmukh et al. ${ }^{[2]}$ and Heuer et al. ${ }^{[3]}$ have described two cases where migrated coils were removed by arteriotomy of the M1 and the angular artery, respectively, after the failed endovascular retrievals. However, stenosis and secondary occlusion are known surgical complications for arteriotomy and repair in microvessels, and this risk might be increased in patients with cerebral vasospasm after SAH. It was also feared that the arteriotomy would tear the distended artery because of the migrated coil. ${ }^{[4]}$ Therefore, moving the migrated coils from less important vessels may be considered to keep the essential vessels intact. Mariak et al. ${ }^{[4]}$ reported a successful case where the migrated coil in the MCA was removed through a small branch of the M2 segment for fear of tearing the MCA by direct M1 arteriotomy.

In our two cases, migrated coils were intraoperatively found to escape from the aneurysm dome. The distended MCA wall looked thin and fragile, and good collateral circulation from the contralateral A1 was confirmed. To avoid the risk of tear and stenosis of the MCA after arterotomy and repair, we decided to remove the coils through the proximal A1 near the ICA bifurcation. Both patients recovered well without any neurological deficits, and the follow-up CT angiogram showed good cross circulation. Without necessity to repair the arteriotomy in emergency, this procedure could be easy and prompt, thereby, reducing the ischemic risk with less time for temporary clipping. For lack of long-term follow up in our cases, we could not draw conclusions about the longterm safety of compromise of the unilateral ACA. Taylor et al. ${ }^{[10]}$ reported 15 years prognosis of 17 patients who had undergone unilateral ACA ligation as the sole treatment for an anterior communicating artery aneurysm. In their series, late ischemia was proved in only one and this occurred in the region of the contralateral MCA. These authors suggested that late ischemia was not a major risk in patients with ligation of the ACA.

In summary, we described our experiences of removing the migrated coils located in the ICA bifurcation and proximal MCA via excised proximal A1, which was found to be effective in our two cases with good results. This surgical procedure might be considered as an alternative route in selected patients with good cross circulation in emergency. In addition, we also have reported a rare occasion of coil migration following stent-assisted coiling, which might have resulted from unsatisfactory wall apposition of the stent due to curved vessel.

\section{References}

1. Thornton J, Dovey Z, Alazzaz A, Misra M, Aletich VA, Debrun GM, et al. Surgery following endovascular coiling of intracranial aneurysms. Surg Neurol 2000;54:352-60.

2. Deshmukh VR, Klopfenstein J, Albuquerque FC, Kim LJ, Spetzler RF. Surgical management of distal coil migration and arterial perforation after attempted coil embolization of a ruptured ophthalmic artery aneurysm: technical case report. Neurosurgery 2006;58:ONS-E379.

3. Heuer GG, Zaghloul KA, Roberts R, Stiefel MF, Storm PB. Successful microsurgical extraction of a migrated coil in a pediatric patient after failed endovascular closure of a Blalock-Taussig shunt: Case report. J Neurosurg 2007;106:136-8.

4. Mariak Z, Kochanowicz J, Kordecki K, Jadeszko M, Lyson T, Lewko J. Surgical evacuation of an embolization coil from the middle cerebral artery. Neurol Neurochir Pol 2004;38:533-7.

5. Chen Z, Zhu G, Feng H, Miao H. Rupture of a saccular microaneurysm of the supraclinoid internal carotid artery after mild head injury in a case with prominent posterior clinoid process. Neurol India 2006;54:451-2.

6. Gao BL, Li MH, Wang YL, Fang C. Delayed coil migration from a small wide-necked aneurysm after stent-assisted embolization: Case report and literature review. Neuroradiology 2006;48:333-7.

7. Luo CB, Chang FC, Teng MM, Guo WY, Chang CY. Stent management of coil herniation in embolization of internal carotid aneurysms. A.JNR Am J Neuroradiol 2008;29:1951-5.

8. Ebrahimi N, Claus B, Lee CY, Biondi A, Benndorf G. Stent conformity in curved vascular models with simulated aneurysm necks using flat-panel CT: An in vitro study. AJNR Am J Neuroradiol 2007;28:823-9.

9. Henkes H, Lowens S, Preiss H, Reinartz J, Miloslavski E, Kuhne D. A new device for endovascular coil retrieval from intracranial vessels: Alligator retrieval device. AJNR Am J Neuroradiol 2006;27:327-9.

10. Taylor W, Miller JD, Todd NV. Long-term outcome following anterior cerebral artery ligation for ruptured anterior communicating artery aneurysms. J Neurosurg 1991;74:51-4.

Accepted on 07-03-2009

Source of Support: Nil, Conflict of Interest: None declared. 